\title{
An Experimental Study on the Endurance of Immunologic Memory of Intradermal Micro-Injection with Rabies Vaccine and Boosting Immune Effect
}

\author{
YuHua Yang ${ }^{1}$ and JinGui Zhang ${ }^{2}$
}

\begin{abstract}
The purpose of the experimental study is to observe the effect of the immune recall responses induced by intramuscular (IM) injection with concentrated Primary Hamster Kidney Cell Vaccine(PHKCV) on days $180,365,545$ and 730 after $0.1 \mathrm{~mL}$ nonconcentrated PHKCV was inoculated intradermally (ID), so as to determine the duration of the immunologic memory induced by ID $0.1 \mathrm{~mL}$ nonconcentrated PHKCV and chieve the goal of protecting the rabies patients with a short incubation. The results indicated that there were four intervals of 180,365 , 545 and 730 days between ID and IM PHKCV, the seroconversion rates being respectively $96.55 \%, 100 \%, 100 \%$ (180 day); $76.67 \%, 100 \%, 100 \%$ (365 day); $75.86 \%, 100 \%, 100 \%$ (545 day) and $75.00 \%, 100 \%, 100 \%$ (730 day) on days 7,14 and 30 after the first dose IM, the seroconversion rates of four experimental groups were all markedly higher than that of five routine doses $(40.74 \%)(P<0.01)$ on days 7 after the first dose IM. The immune endurance of concentrated PHKCV and the enhancing immune effect of the vaccine following booster injection 365 days later were also observed. The results indicated that the seroconversion rates on days 180,365 and 380 (on day 15 after boosting injection) were $96 \%, 83.33 \%$ and $100 \%$ respectively. The stated results prove that the concentrated PHKCV has better immune endurance and the immunologic memory induced by ID $0.1 \mathrm{~mL}$ nonconcentrated PHKCV can maintain two years. The immune effect inoculated by IM two or three doses on day 730 after ID $0.1 \mathrm{~mL}$ nonconcentrated PHKCV was better than that of five routine doses as the first time vaccination. If intradermal micro-injection were performed with higher titre of rabies vaccines, the longer time of immunologic memory would be maintained. J Epidemiol, $1999 ; 9$ : 209-215.
\end{abstract}

concentrated PHKCV, pre-exposure immunization , immunologic memory

Canine rabies, which is a dreaded viral disease with almost $100 \%$ fatality, remains endemic in 106 countries $^{1}$, is responsible for about 60000 human deaths and more than 10 million conducted human postexposure vaccinations worldwide each year $^{2}$. The highest incidence occurs in Asia with above 98 percent of reported human deaths on a global scale ${ }^{1}$. Rabies is widespread in China, covering almost the whole country except one of the northwest provinces, although the incidence rates vary from one province to the next. The total number of human rabies cases was several thousands each year from $1980-1990$,with a death roll of 50000 . The incidence is usually higher in some of the south provinces ${ }^{3}$. Since 1993 , the incidence has declined sharply with the number of the cases limit- ed from 150 to 500 persons each year. Since 1990, the number of dogs has risen markedly in towns and countryside, and the number of persons bitten by dogs has correspondingly increased, so has increased the number of vaccinated persons. As a result, although the incidence has decreased, the number of persons bitten by dogs and then immunized with vaccine has increased, with 5 million human postexposure vaccination in $1996^{1)}$. From the analysis of immunization with PHKCV in the 3 northeast provinces of China, we know 56 cases failed among 50000 cases immunized and there are about 60 percent cases with an incubation period of less than 30 days 4 .

Up to now, although tissue cell culture vaccine has been used for protecting humans bitten by rabid animals at home

Received February 23, 1998 ; accepted February 14, 1999.

${ }^{1}$ Department of Zoonoses, Shandong Provincial Sanitation \& Anti-epidemic Station, Jinan, P.R.China.

${ }^{2}$ Pingyin County Sanitation \& Anti-epidemic Station, Pingyin, Jinan, China.

Address for correspondence : YuHua Yang, 72 Jingshi Road, Jinan, Shandong, P. R. China. 
and abroad for tens years, the incidence of rabies remains after postexposure immunisation ${ }^{59}$ ). In present condition, the people bitten by rabid animals are immunized more and more, however the incidence after postexposure immunization is still causing death due to rabies. We consider the reason is that the whole course of routine five doses needs longer time and it is difficult for the patients with a short incubation to be protected. Therefore, we assume that the intradermal (ID)injection of widespread pre-exposure immunization for high risk group of rabies exposure be conducted so that the human body will be able to produce immunologic memory, and once they are bitten by rabid animals immune recall response may be induced by intramuscular (IM) two or three doses, which can produce a higher antibody level in a short time, with a view to effectively protecting the persons bitten and patients with a short incubation. The purpose of this experimental study is to find out how long it could be to induce immune recall after $\mathrm{ID}$ microinjection with PHKCV, then to provide scientific basis for vaccination to those at high risk of rabies exposure and finally to protect those with a short incubation period. For this reason, we carried out this experimental study. Simulaneously, we observed the effect of the immune endurance and its boosting injection of home-made concentrated PHKCV which was first used in 1995.

\section{MATERIALS AND METHODS}

\section{Subjects}

There were 15 junior middle schools in Pingyin county of Jinan city, we selected one of the largest schools, and there were 923 students distributed in 19 classes of the school. We called students together to hold a meeting and got infomed consent to join this study. 160 volunteers were sampled with method of cluster sampling from those without the history of rabies vaccinaton and being bitten by animals in the school . They were divided into five groups randomly according to their seat numbers. Of the 160 volunteers, only 10 with seroconversion before vaccination and 7 school dropped were rejected. Of the 143 subjects, 72 males and 71 femals, M:F ratio $=1.01: 1$. There was no statistical difference of sex distribution between the groups ( $\chi^{2}=2.83, \mathrm{P}=0.586$ ). They were aged 11-15, with an average age of 13 . There were statistical difference of age distribution between the groups $(F=16.18$, $\mathrm{P}<0.001)$.

\section{Vaccine}

The antirabies $\mathrm{PHKCV}$, liquid $2 \mathrm{~mL}$ per-dose with the nonconcentrated PHKCV lot 940603-2 and concentrated PHKCV lot 9511003-9, 9504036-14 were produced in Changchun Biological Products Institute of Health Ministry of China. The nonconcentrated PHKCV lot 940603-2 was perfomed by $\mathrm{ID} 0.1 \mathrm{~mL}$ in the deltiod region of the arm. The lot
9511003-9 of concentrated PHKCV was performed five routine doses IM in the control group and experimental group 1. The lot 9504036-14 of concentrated PHKCV was performed by IM in the experimental group 2 . The concentrated PHKCV, liquid $2 \mathrm{~mL}$ per-dose with lot $96015-1$ produced in Beijing Biological Products Institute of Health Ministry of China was performed by IM in the experimental group 3 and in the control group of boosting immunization on day 365 . The lot 960212-2 of concentrated PHKCV was performed by IM in the experimental group 4. All of the vaccines were used within the time of efficacy. The potency of concentrated PHKCV was 2.5 international unit (IU) per-dose or more, and that of nonconcentrated PHKCV was less than $2.5 \mathrm{IU}$ perdose.

\section{Observation Time \\ From October 271994 to Novermber 281996.}

\section{Regimen}

See Table 1.

\section{Blood Samples Collection}

Blood samples were collected before vaccination and subsequently on days 7, 14 and 30 after the first dose IM of five routine doses and on days 180,365 and 380 in the control group. The serum was separeted from blood and stored at $18^{\circ} \mathrm{C}$. The serum was measured by antirabies antibody test in batches.

\section{Determination of serum antibody}

The determination of serum antibody was measured with the Enzyme-Linked Immunosorbent Assay (ELISA) ${ }^{10,11}$. The reagent kit was manufacured by Lanzhou Biological Products Institute of Health Ministry of China. Operation of test was performed according to manual of reagent kit. The seroconversion of serum samples was considered positive at $O D$ value equal to or three times above that of $\mathrm{OD}$ value of negative control.

\section{Statistical Analysis}

The seroconversion rates were analysed statistically by $\chi$ square test for comparing five groups and $\chi$ square cut apart for comparing every two groups. All the data were input and analysed with EPINFO programe.

\section{RESULTS}

\section{The seroconversion rates of five groups on days 7,14 and 30 after the first dose IM.}

In order to compare the endurance of immunologic memory of the experimental groups inoculated with ID $0.1 \mathrm{~mL}$ nonconcentrated PHKCV, with four intervals of $180,365,545$ and 
730 days between ID and IM, we observed the immunologic recall responses following IM concentrated on days 7 , 14 and 30, after first dose IM, and compared it with the control group of a routine inoculation with 5 doses of concentrated PHKCV. The results show that the seroconversion rates of four experimental groups on day 7 after the first dose IM were $96.55 \%, 76.67 \%, 75.86 \%$ and $75.00 \%$ respectively, which was significantly higher than that of the control group $(40.74 \%)\left(\chi^{2}=22.99, P=0.001274\right)$. By contrast, the seroconversion rate of five groups on day 14 after the first dose IM also was of no significant difference . The seroconversion rates of five groups on day 30 were con- sistent, and there was no statistical difference in five groups on day 30 . See Table 2 .

2.The comparative analysis of seroconversion rates of every two groups on day 7 after the first dose IM.

According to Table 2, there were significantly statistical differences of seroconversion rates in five groups on day 7 after the first dose IM, so further analyses were performed by method of $\chi$ square cut apart for comparing every two groups. According to findings in Table 3 , there were significantly statistical differences between group No.5 and No.1 or No.2 or No. 3 or No. 4 respectively $(\mathrm{P}<0.01)$, and between group

Table 1. PHKCV regimen.

\begin{tabular}{|c|c|c|c|c|}
\hline Group No. & Schedule & Dosage & Site & Route \\
\hline \multirow{3}{*}{ 1.Experimental 1} & On day 180 before IM & $0.1 \mathrm{~mL}$ & \multirow{3}{*}{ Triceps brachii } & ID \\
\hline & & & & \\
\hline & day $0,3,7,14,30$ & $2 \mathrm{~mL} /$ time & & IM \\
\hline \multirow{3}{*}{ 2.Experimental 2} & On day 365 before IM & $0.1 \mathrm{~mL}$ & \multirow{3}{*}{ Triceps brachii } & ID \\
\hline & & & & \\
\hline & day $0,3,7,14,30$ & $2 \mathrm{~mL} /$ time & & IM \\
\hline \multirow{3}{*}{ 3.Experimental 3} & On day 545 before IM & $0.1 \mathrm{~mL}$ & \multirow{3}{*}{ Triceps brachii } & ID \\
\hline & & & & \\
\hline & day $0,3,7,14,30$ & $2 \mathrm{~mL} /$ time & & IM \\
\hline \multirow[b]{2}{*}{ 4.Experimental 4} & On day 730 before IM & $0.1 \mathrm{~mL}$ & \multirow[b]{2}{*}{ Triceps brachii } & ID \\
\hline & day $0,3,7,14,30$ & $2 \mathrm{~mL} /$ time & & IM \\
\hline 5.Control & day $0,3,7,14,30$ & $2 \mathrm{~mL} /$ time & Triceps brachii & IM \\
\hline
\end{tabular}

Table 2. Seroconversion rates of five groups in different period.

\begin{tabular}{|c|c|c|c|c|c|c|}
\hline \multirow{2}{*}{$\begin{array}{l}\text { Group } \\
\text { No. }\end{array}$} & \multicolumn{2}{|c|}{ Day 7} & \multicolumn{2}{|c|}{ Day 14} & \multicolumn{2}{|c|}{ Day 30} \\
\hline & $\begin{array}{l}\text { Positive } \\
\text { Rate }(\%)\end{array}$ & $\begin{array}{c}95 \% \mathrm{Cl}^{*} \\
(\%)\end{array}$ & $\begin{array}{l}\text { Positive } \\
\text { Rate(\%) }\end{array}$ & $\begin{array}{c}95 \% \mathrm{CI}^{*} \\
(\%)\end{array}$ & $\begin{array}{l}\text { Positive } \\
\text { Rate }(\%)\end{array}$ & $\begin{array}{c}95 \% \mathrm{CI}^{*} \\
(\%)\end{array}$ \\
\hline 1 & $28 / 29(96.55)$ & $(82-100)$ & $30 / 30(100.0)$ & $(88-100)$ & $30 / 30(100.00)$ & $(88-100)$ \\
\hline 2 & $23 / 30(76.67)$ & $(58-90)$ & $31 / 31(100.0)$ & $(89-100)$ & $31 / 31(100.00)$ & $(89-100)$ \\
\hline 3 & $22 / 29(75.86)$ & $(56-90)$ & $30 / 30(100.0)$ & $(88-100)$ & $28 / 28(100.00)$ & $(88-100)$ \\
\hline 4 & $18 / 24(75.00)$ & $(53-90)$ & $24 / 24(100.0)$ & $(86-100)$ & $24 / 24(100.00)$ & $(86-100)$ \\
\hline 5 & $11 / 27(40.74)$ & $(22-61)$ & $24 / 27(88.89)$ & $(71-98)$ & $27 / 27(100.00)$ & $(87-100)$ \\
\hline Total & $102 / 139(73.38)$ & $(66-81)$ & $133 / 142(97.89)$ & $(96-100)$ & $140 / 140(100.0)$ & \\
\hline $\begin{array}{l}\chi^{2} \text { value } \\
\mathrm{P} \text { value }\end{array}$ & \multicolumn{2}{|c|}{$\begin{array}{c}22.99 \\
0001274\end{array}$} & \multicolumn{2}{|c|}{$0.810^{* *}$} & \multicolumn{2}{|l|}{$* * *$} \\
\hline
\end{tabular}

* The calculation of $95 \% \mathrm{CI}$ of positive rates in the groups was consulting related literature. ${ }^{12}$

** The calculation was analysed by Ridit, then the $\chi^{2}$ value was calculated. ${ }^{13}$

*** Because the data in the same row are zero, the $\chi^{2}$ value can not be calculated. 
No.land No.2 or No.3 group ( $\mathrm{P}<0.05)$. There was no statistical difference between group No.2 and No.3 or No.4.

3.The immune endurance of routine vaccination with concentrated PHKCV and its effect of boosting immunization.

Because the concentrated PHKCV was first used in China in 1995, the immune effect of the vaccine was unknown before this study. In order to investigate the immune endurance and the immune effect following boosting immunization, we made a further research on the control group. It can be seen from Table 4 that both the seroconversion rates on day 180 and 365 after the first dose IM and on day 15 after boosting immunization were high, which proved that the immune effect with concentrated PHKCV was related to endurance. The seroconversion rate on day 15 after boosting a dose was all positive.

During the observation, only one of the subjects had erythema, rash and scratch on the limbs and trunk, within 24 hours after treatment in the hospital the eythema and rash disappeared.

\section{DISCUSSION}

Regarding the five routine doses IM as control group in the experimental study, and taking the IM concentrated PHKCV on days $180,365,545$ and 730 after ID $0.1 \mathrm{~mL}$ nonconcentrated PHKCV as experimental groups, the results indicated that the immune recall responses could be induced by inoculation with concentrated PHKCV on days $180,365,545$ and 730 after ID $0.1 \mathrm{~mL}$ nonconcentrated PHKCV, and after vaccinating with two or three doses (from 7 th day to 14 th day), the seroconversion rate could achieve $75 \%-100 \%$, however, that of the control group is only from $40.74 \%$ to $88.89 \%$. According to related literature reported, while a high peak of the seroconversion rate of antibody occured, the antibody level would be at a high peak ${ }^{16)}$, and in another literature reported by the same auther, the antibody level of experimental groups was significantly higher than that of the control group as well ${ }^{17}$. This indicated that utilizing the immune response could create a high antibody reaction in a short time.

Since rabies is a kind of lethal infectious disease, if one develops it, he will die. Besides postexposure immunization is

Table 3. Comparative analysis of seroconversion rates of every two groups on day 7 after the first dose IM.

\begin{tabular}{|c|c|c|c|c|c|}
\hline \multirow{2}{*}{ Group-Group } & \multirow{2}{*}{$\chi^{2}$ value } & \multicolumn{2}{|c|}{$\chi^{2}$ Limit value* } & \multirow{2}{*}{$\begin{array}{l}95 \% \mathrm{CI} \text { of difference of positive } * * \\
\text { rates between every two groups }(\%)\end{array}$} & \multirow{2}{*}{$P$ value } \\
\hline & & $P=0.05$ & $P=0.01$ & & \\
\hline $4-5$ & 6.08 & 1.29 & 2.54 & $8.89-59.63$ & $<0.01$ \\
\hline $3-5$ & 7.13 & 2.69 & 4.37 & $10.91-59.33$ & $<0.01$ \\
\hline $2-5$ & 7.62 & 4.52 & 6.72 & $11.90-59.86$ & $<0.01$ \\
\hline $1-5$ & 18.05 & 7.42 & 10.51 & $36.12-75.50$ & $<0.01$ \\
\hline $3-4$ & 0.01 & 1.29 & 2.54 & $-22.44-24.16$ & $>0.05$ \\
\hline $2-4$ & 0.02 & 2.69 & 4.37 & $-21.33-24.67$ & $>0.05$ \\
\hline $1-4$ & 3.61 & 4.52 & 6.72 & $3.00-40.10$ & $>0.05$ \\
\hline $2-3$ & 0.01 & 1.29 & 2.54 & $-20.91-21.72$ & $>0.05$ \\
\hline $1-3$ & 3.63 & 2.69 & 4.37 & $3.76-37.62$ & $<0.05$ \\
\hline $1-2$ & 3.43 & 1.29 & 2.54 & $3.35-36.41$ & $<0.01$ \\
\hline
\end{tabular}

Note: $1^{*}$.According to the positive rates arranged in rates from small to large order, and comparative analysis of the rates of every two groups were performed. ${ }^{14}$ $2 * *$.The $95 \% \mathrm{CI}$ of differences of positive rates between two groups were calculated according to related literature. ${ }^{15}$

Table 4. Seroconversion rates in different period after immunization with concentrated PHKCV.

\begin{tabular}{lccccccc}
\hline & Before & \multicolumn{5}{c}{ After Immunization } \\
\cline { 3 - 7 } & Immunization & Day 7 & Day 14 & Day 30 & Day 180 & Day 365 & Day 380 \\
\hline Positive & $0 / 27$ & $11 / 27$ & $24 / 27$ & $27 / 27$ & $24 / 25$ & $20 / 24$ & $22 / 22$ \\
Rates (\%) & 0.00 & 40.74 & 88.89 & 100.00 & 96.00 & 83.33 & 100.00 \\
$95 \%$ CI (\%) & $0-13$ & $22-61$ & $71-98$ & $87-100$ & $80-100$ & $63-95$ & $85-100$ \\
\hline
\end{tabular}


a treatment inoculation, its immune effect relates to the life and death of patients. Thus, utilizing the characteristics of the immune recall response to protect the patients with a short incubation, and to decrease the death roll is of important significance. However, because of the difference of the lethality, incubation and vaccines of the other infectious diseases and the preventive inoculation, the vaccines of the first time may be preventive. Thereby, the principle of recall responses for most infectious diseases application is of little significance, compared with rabies.

Concerning pre-exposure immunization, the protective antibody titre may maintain from one year and a half to two years with Human Diploid Cell Vaccine (HDCV) intradermal $0.1 \mathrm{~mL}$ three times abroad, and few can maintain six years ${ }^{18)}$ .It is emphasized abroad that a certain protective antibody level of pre-exposure immunization should be maintained. This study, however, proposed that the pre-exposure immunization merely maintain immunologic memory, instead of protective antibody level. As a result of the endurance time of the immunologic memory would remain longer than that of maintaining protective antibody level. Like this, the times or dosages of pre-exposure immunization would be reduced. Since this viewpoint is put forward, the pre-exposure immunization can be developed in the practice and it is easy and feasibe to extend the application. Meanwhile, it is neither economical nor convenient that more times or dosages of vaccination are performed before humans are bitten by rabid animals, and especially it is uneasy to be accepted by people in the developing countries ${ }^{19,20)}$. The pre-exposure immunization should be regarded as auxiliary, the postexposure immunization taken as principal. Because the immunologic memory caused by ID micro-injection of pre-exposure immunization induces the immune recall response of postexposure immunization and a higher antibody level in a short time (within 7 days), it is significant for the patients with a short incubation. Since B cell of the humoral immunity is the same as $\mathrm{T}$ cell of the cellular immunity in the way of immunologic memory function, and the lifespan of T cell is longer than that of B cell, a few years longer, the immunologic memory of cellular immunity induced by above intradermal micro-injection with PHKCV would be ever-lasting immuologic memory. In immunologic memory, $\mathrm{T}$ cell determines the extent of immunologic memory, while $B$ cell determines the quality of immunologic memory ${ }^{21)}$. In addition, the unity and steadiness of viral antigen is also an important factor to decide the endurance of immunologic memory ${ }^{22)}$. However, rabies virus is just a kind of more conservative virus with relatively few variations. Its glycoprotein has relatively more variation than that of the other protiens of rabies virus. The homology of the nucleic acid of the viral glycoprotein is above 80 percent in the different strains of rabies virus ${ }^{23}$. However, the homology of the nucleic acid of the other proteins such as nucleoprotein, phosphoprotein, matrix protein and RNA polymerase is even higher in different strains of rabies virus ${ }^{24)}$. For this reason, intradermal micro-injection with PHKCV would make the endurance of immunologic memory last longer, not just 2 years of immunologic memory as proved in this experimental study. Because of limited condition in this study, the vaccine used by intradermal micro-injection was nonconcentrated $\mathrm{PHKCV}$, if we used intradermal micro-injection of pre-exposure with concentrated PHKCV or other higher potency vaccine, the time of immunologic memory would maintain even longer. Even though pre-exposure inoculation was three doses overseas, and would boost a dose after being bitten by rabid animals, in China and the other developing contries for the reason of economic level and other factors, its feasility in practice, would not be achieved. The pre-exposure immunization is only once with micro-injection of PHKCV and merely maintains immunologic memory, which otherwise maintain even longer time of immunologic memory.

Compared with the three doses of pre-exposure immunisation in a month and boosting a dose after being bitten by rabid animals abroad, in spite of the same doses, all being four times, the doses of pre-exposure immunization in this study were less than that of pre-exposure immunisation overseas, which was even easier to be received by people. Compared with routine five doses of postexposure immunization domestically, the dose of immunization was not only one dose less, but also the less dosage of pre-exposure immmunisation, and the immune effect was more effictive than that of routine five doses of postexposure immunization at first time. The method of intradermally inoculating 20 persons with one dosage of $\mathrm{PHKCV}$ is simple and convenient. Thereby, it may be used largely and especially performed in the high risk group, such as venterinarins, experimental workers, forest workers, rural postmen, butchers and the children in contact with animals or dog-keepers and it is worth practicing.

According to related literature reported, the antibody level of immune recall response and seroconversion rates could also reach a high peak from 7 days to 14 days after the first dose IM with concentrated PHKCV, which is significantly higher than that of nonconcentrated PHKCV $(\mathrm{P}<0.01)^{1 \text { th }}$. The peak of seroconversion rates of concentrated $\mathrm{PHKCV}$ and nonconcentrated PHKCV are on days 30 and 45 respectively ${ }^{17,25)}$. The results of the experimental study indicated that the seroconversion rates on days 180 and 365 after vaccination were relatively high, with $96 \%$ and $83.33 \%$ respectively, which is much higher than that of nonconcentrated PHKCV ${ }^{26)}$. The seroconversion rates on days 180 and 365 after vaccination with nonconcentrated PHKCV came down about 50\% and dropped to $30.50 \%$ respectively ${ }^{27}$. Thus it can be seen that the concentrated PHKCV used in China since 1995 has relatively better immune endurance, and seroconversion rate on day 15 after boosting immunization is $100 \%$. The intradermal micro-injec- 
tion of pre-exposure immunization with PHKCVand intramuscular injection was performed after being bitten by rabid animals, creates higher seroconversion rates from day 7 to day 365 after the first dose IM, and has the important significance for protecting patients with different incubation. Moreover, common incubation of rabies is from 15 days to 60 days ${ }^{28}$, which can be completely covered by protective period of the experimental study.

The shortest incubation of rabies is 6 days, but the antirabies antibody can achieve protective antibody level from 10 days to 14 days after vaccination. In the ten days among vaccination and produced antibody and the protective level of antirabies antibody, the immunity of body is a blank without antirabies antibody or lowed antibody level. Vaccine and antiserum used at the same time forms the linking up of the passive immunization and active immunization, which can increase the immune effect ${ }^{29)}$. Because of intradermal micro-injection of pre-exposure immunization, it might induce a quick and high immune recall response and a higher antibody level, makes up for the blank of ten days and avoids the passive immunization. And yet passive immunization is transient and limited. Moreover, the products of passive immunization are expensive and are not used universally in countryside of China. More importantly there are severe side-effects after the horse serum of antirabies is used.

Apart from the difference of the seroconversion rates on day 7 after the first dose IM between the group No.1 and No.2 being about $20 \%$, the difference of that between the group No.2, No.3 and No.4 was small, which might relate to potency of PHKCV and originate from the lowed constant level of immunologic memory ${ }^{18,30)}$.

As for the side-effect of rabies vaccine, endotoxin content of rabies vaccine for humans after concentrated is increased markedly and induces the side-effect after vaccination ${ }^{31}$. Of the 143 subjects, only one had the side-effect such as erythema, rush and scratch and so on.

\section{REFERENCES}

1. WHO, Division of emerging and other communicable diseases surveillance and control. World survey of rabies No.32 for the year 1996. 1998:2-6.

2. Meslin FX, Stohr K. Prospects for immunization against rabies in developing countries. In: Dodet $\mathrm{B}$, Meslin FX,eds. Rabies control in Asia. Elsevier, Paris ,1997:1518.

3. Tang $Q$, Zhao $X Q$, Dou Z. Human epidemiology and risk factors for rabies. In: Dodet $b$, Meslin FX,eds. Rabies control in Asia: Country reports. Elsevier, Paris, 1997:130-136.

4. Ren CJ. Primary Hamster Kidney Cell Vaccine in China. In: Thraenhart O., Kopronski H., Bogel K., Sureau P., eds. Proceeding of the 2 nd international IMVI ESSEN/WHO symposium. Essen 5-7, July 1988 and report of the WHO consultation on rabies. 1989.

5. Wilde H., Sirkawin S., Sabcharoen A., Kingnate D., Tantawichien T., Harischandra P.A. L., Chaiyabutr N., Silva D.G.H.de, Fernando L., Liyanage J. B. , Sitprija V. .Failure of postexposure treatment of rabies in children. Clin Infect Dis, 1996;22:228-238.

6. Wilde H., Choomkasien P., Hemachudha T. , Supich C., Chutivongse S. . Failure of rabies postexposure treatment in Thailand. Vaccine, 1989;7:49-52.

7. Xia X. Z., Wang J. L. Analysis of failure causes of preventing rabies with humans rabies adjuvant vaccine of 16 cases. Chin J Epidmiol, 1988;9:383-384.

8. Qiu Y.H. Report on immune failure and death of intramuscular rabies vaccine of 3 cases. Chin J Zoonoses, 1991;7(6):41.

9. Kuresishi A., Xu L.Z., Wu H., Stiver H.G. . Rabies in China: recommendations for control. Bull WHO, 1992; 70: 443-450.

10. Zeng M, Wang YL, Liang Y, Li G.M.. Comparative study of ELISA and NIH on antigen-detecting of rabies vaccine. Prog Microbiol Immunol, 1996;24(3):20-22.

11. Wen Z.Q., Yan S.Q., Meng Z.Y., Zhang W.X. . Rabies vaccine potency test with parallel line bioassay method by ELISA. Chin J Microbiol Immunol, 1996;16(2):149152.

12. Jin P.H., Zhan S.K., Zhang Z.H. In: Medical Statistical Methods. Shanghai Medical University Publishing House, Shanghai, 1993: 470-471.

13. Jin P.H., Zhan S.K., Zhang Z.H. In:Medical Statistical Methods. Shanghai Medical University Publishing House, Shanghai, 1993:244-245.

14. Luo W.H., Wang J., Hao E.Z., Gao Y., Zhu B.T., Zhou Y.X., Wan Q.Y., Xu J. H.,Lei G.H.. Study on significant limited value of $\chi$ square test of comparing every two samples rates of multiple sample rate. Chin J Health Statistics, 1995;12(1):42-43.

15. Yang W.X., Yang J.Z.. Applying reliable interval to medical study and its article. Chin Public Health, 1990;6(10):457-459.

16. Yang Y.H., Zhang J.G., Huang J.T., Liu C.X., Tao X.R., Chen W. Observation on effect of regarding an intradermal inoculation of rabies vaccine as foundational immunization. Chin J Zoonoses, 1995;11(2):51-53,14.

17. Yang Y.H., Tao X.R, Zhang J.G., Su J.Y., Cui S., Zheng D.M. . Observation on the immune effect of concentrated rabies vaccine for humans. Chin Public Health, 1996;12 (9):201-202.

18. Briggs D.J., Schwenke J.R.. Longevity of rabies antibody titre in recipients of human diploid cell rabies vaccine. Vaccine, 1992;10:125-129. 
19. Kenneth W., Bernard, Daniel B., Fishbein. Pre-exposure rabies prophylaxis for travellers: are the benefits worth the cost? Vaccine, 1991;9:833-836.

20. Warrell MJ, Nicholson KG, Warrell DA, Sautharasamai P, Chanthavanich P, Viravan C, Sinhaseni A, Chiewbambroongkiat MK, Pouradier-Duteil X, Xueref C, Phanfung R, Udomsakdi D. Economical multiple-site intradermal immunization with human diploid-cell-strain vaccine is effective for post-exposure rabies prophylaxis. Lancent, 1985;8437: 1059-1062.

21. Bai R.D. In:Immunology. Shandong Science and Technology Publishing House, Jinan, 1984: 217-221

22. Shanghai Second Medical College. In: Medical Microbiology. People's Health Publishing House, Beijing, 1979:279-280.

23. Wunner W.H., Larson J.K., Dietzchold B, Smith C.L.. The molecular biology of rabies virus. Rev Infect Dis, 1988;10(sup 4):s771-s784.

24. Yang Y.H., Wang J.X.. Recent developments on research of molecular biology of rabies virus. J Prev Med Inf. 1994;10( 3):148 -152.

25. Li X.X., Hu L.W., Wang R.H., Cai C.M.. Obsanervation on the serological effects of preventive vaccination with primary hamster kidney tissue culture rabies vaccine. Chin J Epidemiol, 1984;5(6):216-218.
26. Yang Y.H., Su J.Y., Cui S., Zheng D.M., Huang J.T., Tao X.R.. An experimental study on different immune methods of rabies vaccine. Modern Prev Med, 1996;23 (3): 149-151,157.

27. Li Y.Z., Gan Y., Li P., Zheng S.H., Wang S.B. Wang D.S., Deng J., Wang H.L., Ren Y.P.,Zhou X.L.. Observation on the antibodies continuity after immunization with rabies vaccine and effect of the enhancing immunization. Chin J Zoonoses, 1993;9(3):37-38.

28. Atfred $S$ Evans. Viral Infectious of HumansEpidemiology and Control. People's Health Publishing House, Beijing, 1984: 12-15.

29. Lu J.H., Zhang Y.H., Zhao K.. In: Biologicals in Medicine. Beijing: People's Health Publishing House, 1995:565.

30. Strady A., Lang J.,Lienard M., Blondeau C., Jaussaud $R$., Plotkin Stanley AP. Antibody persistence following preexposure regimens of cell culture rabies vaccines: 10year follow-up and proposal for a new booster policy. J Infect Dis, 1998;177:1290-1295.

31. Li G.Y., Xie X.M., Xi L., Feng D.J.. Study of endotoxin content in concentrated and unconcentrated rabies vaccine for humans use. Prog Microbiol Immunol, 1996;24(2):24-27. 